\title{
The American Society of Breast Surgeons and Quality Payment Programs: Ranking, Defining, and Benchmarking More Than 1 Million Patient Quality Measure Encounters
}

\author{
Jeffrey Landercasper, $\mathrm{MD}^{1}$, Lisa Bailey, $\mathrm{MD}^{2}$, Robert Buras, $\mathrm{MD}^{3}$, Ed Clifford, $\mathrm{MD}^{4}$, Amy C. Degnim, $\mathrm{MD}^{5}$, \\ Leila Thanasoulis, $\mathrm{MD}^{6}$, Oluwadamilola M. Fayanju, $\mathrm{MD}^{7}$, Judy A. Tjoe, $\mathrm{MD}^{8}$, and Roshni Rao, MD \\ ${ }^{1}$ Gundersen Health System, La Crosse, WI; ${ }^{2}$ Bay Area Breast Surgeons, Inc., Oakland, CA; ${ }^{3}$ Anne Arundel Medical \\ Center, Annapolis, MD; ${ }^{4}$ Baylor Scott and White Healthcare, Dallas, TX; ${ }^{5}$ Mayo Clinic, Rochester, MN; ${ }^{6}$ New York \\ Community Hospital of Brooklyn, Brooklyn, NY; ${ }^{7}$ Duke University, Durham, NC; ${ }^{8}$ Aurora Health Care, Milwaukee, WI; \\ ${ }^{9}$ University of Texas Southwestern Medical Center, Dallas, TX
}

\begin{abstract}
Background. To identify and remediate gaps in the quality of surgical care, the American Society of Breast Surgeons (ASBrS) developed surgeon-specific quality measures (QMs), built a patient registry, and nominated itself to become a Center for Medicare and Medicaid Services (CMS) Qualified Clinical Data Registry (QCDR), thereby linking surgical performance to potential reimbursement and public reporting. This report provides a summary of the program development.

Methods. Using a modified Delphi process, more than 100 measures of care quality were ranked. In compliance with CMS rules, selected QMs were specified with inclusion, exclusion, and exception criteria, then incorporated into an electronic patient registry. After surgeons entered QM data into the registry, the ASBrS provided real-time peer performance comparisons.

Results. After ranking, 9 of 144 measures of quality were chosen, submitted, and subsequently accepted by CMS as a QCDR in 2014. The measures selected were diagnosis of cancer by needle biopsy, surgical-site infection, mastectomy reoperation rate, and appropriateness of specimen imaging, intraoperative specimen orientation, sentinel node use, hereditary assessment, antibiotic choice, and antibiotic duration. More than 1 million patient-measure encounters were captured from 2010 to 2015. Benchmarking
\end{abstract}

(C) The Author(s) 2017. This article is an open access publication

First Received: 13 April 2017;

Published Online: 1 August 2017

J. Landercasper, MD

e-mail: jlanderc@gundersenhealth.org functionality with peer performance comparison was successful. In 2016, the ASBrS provided public transparency on its website for the 2015 performance reported by our surgeon participants.

Conclusions. In an effort to improve quality of care and to participate in CMS quality payment programs, the ASBrS defined QMs, tracked compliance, provided benchmarking, and reported breast-specific QMs to the public.

For more than two decades, strong evidence has indicated variation in the quality of cancer care in the United States. ${ }^{1-19}$ As a result, measurements and audits are necessary to search for gaps in the quality of care. Toward this end, multiple professional organizations have developed condition-specific quality measures (QMs) to assess the clinical performance surrounding the patient-provider encounter.

Quantification of performance can identify variation and opportunities for improvement. If performance assessment is followed by performance comparison among peers (i.e., benchmarking) coupled with transparency among providers, physicians who find themselves in the lower tiers of performance can be motivated to improve, ultimately yielding better overall care at the population level, a phenomenon that recently has been reviewed and demonstrated by several programs. ${ }^{20-26}$

This report aims to describe how the American Society of Breast Surgeons (ASBrS) ranked and defined measures of quality of care and subsequently provided benchmarking functionality for its members to compare their performances with each other. By separate investigations, the actual performance demonstrated by our ASBrS membership for compliance with nine breast surgeon-specific QMs are reported. 
Founded in 1995, the ASBrS is a young organization. Yet, within 20 years, membership has grown to more than 3000 members from more than 50 countries. A decade ago, the Mastery of Breast Surgery Program (referred to as "Mastery" in this report) was created as a patient registry to collect quality measurement data for its members. ${ }^{27}$

Past President Eric Whitacre, who actually programmed Mastery's original electronic patient registry with his son Thomas, understood that "quality measures, in their mature form, did not merely serve as a yardstick of performance, but were a mechanism to help improve quality." 28,29 Armed with this understanding, the ASBrS integrated benchmarking functionality into Mastery, thus aligning the organization with the contemporary principles of optimizing cancer care quality as described by policy stakeholders. ${ }^{2,19,25,30}$

In 2010, Mastery was accepted as a Center for Medicaid and Medicare Services (CMS) Physicians Qualified Reporting Service (PQRS) and then as a Qualified Clinical Data Registry (QCDR) in 2014, linking provider performance to government reimbursement and public reporting. ${ }^{31}$ Surgeons who successfully participated in Mastery in 2016 will avoid the 2018 CMS "payment adjustment" ( $2 \%$ penalty), a further step toward incentivizing performance improvement in tangible ways.

\section{METHODS}

\section{Institutional Review Board}

De-identified QM data were obtained with permission from the ASBrS for the years 2011-2015. The Institutional Review Board (IRB) of the Gundersen Health System deemed the study was not human subjects' research. The need for IRB approval was waived.

\section{Choosing, Defining, and Vetting QM}

From 2009 to 2016, the Patient Safety and Quality Committee (PSQC) of the ASBrS solicited QM domains from its members and reviewed those of other professional organizations. ${ }^{32-39}$ As a result, as early as 2010 , a list of more than 100 domains of quality had been collected, covering all the categories of the Donabedian trilogy (structure, process, and outcomes) and the National Quality Strategy (safety, effectiveness, efficiency, population health, care communication/coordination, patient-centered experience) ${ }^{40,41}$ By 2013, a list of 144 measures underwent three rounds of modified Delphi process ranking by eight members of the PSQC, using a RAND/UCLA Appropriateness Methodology, which replicated an American College of Surgeons effort to rank melanoma measures and was consistent with the National Quality Forum's guide to QM development ${ }^{42,43}$ (Tables 1, 2). During the ranking, quality domains were assigned a score of 1 (not valid) to 9 (valid), with a score of 5 denoting uncertain/equivocal validity. After each round of ranking, the results were discussed within the PSQC by email and phone conferences. At this time, arguments were presented for and against a QM and its rank. A QM was deemed valid if $90 \%$ of the rankings were in the range of seven to nine.

After three rounds of ranking ending in December 2013, nine of the highest ranked measures were "specified" as described and required by $\mathrm{CMS}^{44}$ (Table 3 ). Briefly, exclusions to QM reporting were never included in the performance numerator or denominator. Exceptions were episodes in which performance for a given QM was not met but there was a justifiable reason why that was the case. If so, then the encounter, similar to an exclusion, was not included in the surgeon's performance rate. If an encounter met performance criteria despite typically meeting exception criteria, the encounter was included in the performance rate. Per CMS rules, each QM was linked to a National Quality Strategy Aim and Domain (Table 3). The QMs also were assigned to a Donabedian category and to one or more of the Institute for Healthcare Improvement's "triple aims." 40,45

Each of our QMs underwent vetting in our electronic patient registry (Mastery) by a workgroup before submission to CMS. During this surveillance, a QM was modified, retired, or advanced to the QCDR program based on member input and ASBrS Executive Committee decisions.

\section{Patient Encounters}

To calculate the total number of provider-patient-measure encounters captured in Mastery, we summed the total reports for each individual QM for all study years and all providers who entered data.

\section{Benchmarking}

Each surgeon who entered data into Mastery was able to compare his or her up-to-date performance with the aggregate performance of all other participating surgeons (Fig. 1). The surgeons were not able to access the performance metrics of any other named surgeon or facility.

\section{Data Validation}

In compliance with CMS rules, a data validation strategy was performed annually. A blinded random selection of at least 3\% of QCDR surgeon participants was conducted. After surgeons were selected for review, the ASBrS requested that they send the ASBrS electronic and/or paper 
TABLE 1 Instructions of the American Society of Breast Surgeons for ranking of quality measure domains

\section{Ranking $^{42,43}$}

1. [Evaluate the quality domains] for appropriateness (median ranking) and agreement (dispersion of rankings) to generate quality indicators

2. A measure [will be] considered valid if adherence with this measure is critical to provide quality care to patients with [breast cancer], regardless of cost or feasibility of implementation. Not providing the level of care addressed in the measure would be considered a breach in practice and an indication of unacceptable care

3. Validity rankings are based on the panelists' own personal judgments and not on what they thought other experts believed

4. The measures should apply to the average patient who presents to the average physician at an average hospital

Importance criteria ${ }^{57}$

1. Variation of care

2. Feasibility of measurement, without undue burden

3. Usability for accountability [public transparency or quality payment programs]

4. Applicability for quality improvement activity

Scoring criteria $^{42,43}$

$1=$ not valid

$5=$ uncertain/equivocal validity

$9=$ valid

Verbatim instructions from an American College of Surgeons ranking study ${ }^{43}$

records to verify that their office/hospital records supported the performance "met" and "not met" categories that they had previously reported to the ASBrS via the Mastery registry.

\section{RESULTS}

\section{Hierarchical Order and CMS QCDR Choices}

The median ranking scores for 144 potential QMs ranged from 2 to 9 (Table 2). The nine QMs chosen and their ranking scores were appropriate use of preoperative needle biopsy (9.0), sentinel node surgery (9.0), specimen imaging (9.0), specimen orientation (9.0), hereditary assessment (7.0), mastectomy reoperation rate (7.0), preoperative antibiotics (7.0), antibiotic duration (7.0), and surgical-site infection (SSI) (6.0). The specifications for these QMs are presented in Table 3. The mastectomy reoperation rate and SSI are outcome measures, whereas the remainder are process of care measures.

\section{QM Encounters Captured}

A total of $1,286,011$ unique provider-patient-measure encounters were captured in Mastery during 2011-2015 for the nine QCDR QMs. Performance metrics and trends for each $\mathrm{QM}$ are reported separately.

\section{Data Validation}

The QM reporting rate of inaccuracy by surgeons participating in the 2016 QCDR data validation study of the 2015 Mastery data files was $0.82 \%$ (27 errors in 3285 audited patient-measure encounters). Subsequent reconciliation of discordance between surgeon QM reporting and patient clinical data occurred by communication between the ASBrS and the reporting provider.

\section{CMS Acceptance and Public Transparency}

The Center for Medicare and Medicaid Services accepted the ASBrS QM submitted to them for PQRS participation in 2010-2013 and for QCDR in 2014-2016. In 2016, they discontinued the specimen orientation measure for future reporting and recommended further review of the mastectomy reoperation rate measure. Public reporting of 2015 individual surgeon QCDR data was posted in 2016 on the ASBrS website.

\section{Security}

To our knowledge, no breaches have occurred with any surgeon-user of Mastery identifying the performance of any other surgeon or the identity of any other surgeon's patients. In addition, no breaches by external sources have occurred within the site or during transmission of data to CMS.

\section{DISCUSSION}

\section{Modified Delphi Ranking of QM}

To provide relevant QM for our members, the PSQC of the ASBrS completed a hierarchal ranking of more than 100 candidate measures and narrowed the collection of QMs to fewer than a dozen using accepted methods. ${ }^{42,43}$ Although 
TABLE 2 Hierarchy of quality domains for breast surgeons after the 3rd round of modified Delphi ranking

Quality domain $\quad{\text { Median Validity }{ }^{\mathrm{b}} \text { Agreement }}^{\mathrm{c}}$
score $^{\mathrm{a}}$

Patients receiving diagnosis of cancer by needle biopsy

9

Patients undergoing a formal patient-side-site-procedure verification procedure in the operating room 9

Percentage of cancer patients with orientation of lumpectomy specimen

Clinical stages 1 and 2 node-negative patients offered sentinel lymph node (SLN) surgery

Mastectomy patients with $\geq 4$ positive nodes referred to radiation oncologist

Stages 1, 2, and 3 patients undergoing initial breast cancer surgery with documentation of ER, PR receptor 9 status

Stage 1, 2, and 3 undergoing initial breast cancer surgery with documentation of HER2 neu status

Breast conservation therapy (BCT) patients referred to radiation oncology

Percentage of patients undergoing neoadjuvant therapy before planned breast conservation surgery (BCS) 9 who have imaging marker clip placed in breast

Percentage of patients undergoing lumpectomy for non-palpable cancer with specimen imaging performed

Patients with concordance assessment (testing) of Exam-Imaging-Path by care provider

Patients undergoing breast cancer surgery with final path report indicating largest single tumor size

Patient's compliant with National Quality Forum Quality Measures (NQF QM) for endocrine therapy in 8.5
hormonal receptor positive patients

Trastuzumab is considered or administered within 4 months (120 days) after diagnosis for stage 1, 2, or 38.5 breast cancer that is HER2-positive

Documentation of mastectomy patients offered referral to plastic surgery

Documentation of eligibility of BCT and eligible patients offered BCT

Patients with documentation of patient options for treatment regardless of procedure type

Percentage of patients undergoing BCT with a final ink-negative margin, regardless of number of operations

Patients with adequate history by care provider

Patients with documentation of postoperative cancer staging (AJCC)

Patient's compliant with NQF QM for radiation after lumpectomy

Patients with documentation preoperative (pretreatment) AJCC clinical staging

NCCN compliance with radiation guidelines

Mastectomy patients receiving preoperative antibiotics

Patients with NCCN guideline compliant care for "high risk lesions" identified on needle biopsy (ADH, 8 ALH, FEA, LCIS, papillary lesion, radial scar, mucin-containing lesion)

Patients with NCCN guidelines compliant care for diagnostic evaluation of breast lump 8

Patients with NCCN compliance for postoperative lab imaging, biomarkers in stages 0,1 , and 2 patients 8

NCCN guideline compliance for genetic testing among patients with newly diagnosed breast cancer 8

NCCN guideline compliance for genetics assessment/referral among patients with newly diagnosed breast 8 cancer

Patients with adequate examination by care provider

Patients with final pathologic size $\geq$ stage 1 T1cN0M0 who have documentation of discussion regarding 7.5 adjuvant treatment

Documentation of reason why patient is not eligible for BCT

Patients with adequate review of imagining by care provider

Patients with inflammatory or locally advanced breast cancer who undergo neoadjuvant treatment before 7.5 surgery

High-risk patients with estimated lifetime risk $>20 \%$ offered screening MRI

NCCN compliance for medical oncology recommendations

Risk adjusted re-excision lumpectomy rate after breast-conserving therapy

NCCN guideline compliance for inflammatory breast cancer

NCCN guideline compliance for breast cancer in pregnancy

Patients with predicted estimate of BRCA mutation $>10 \%$ offered BRCA testing

9 Yes Agreement

No Agreement

9 Yes

Agreement

Yes

Agreement

Yes Agreement

No Agreement

No Agreement

Yes Agreement

Yes Agreement

Yes Agreement

No Agreement

No Agreement

Yes Agreement

No Agreement

Yes Agreement

Yes Agreement

Yes Agreement

No Agreement

No Agreement

Yes Agreement

No Agreement

Yes Agreement

No Agreement

Yes Agreement

No Agreement

No Agreement

No Agreement

No Agreement

No Agreement

No Agreement

Yes Agreement

No Indeterminant

No Indeterminant

No Agreement

No Indeterminant

No Indeterminant

Yes Agreement

No Indeterminant

No Indeterminant

No Indeterminant 
TABLE 2 continued

Quality domain Median Validity ${ }^{\mathrm{b}}$ Agreement $^{\mathrm{c}}$ score $^{\mathrm{a}}$

High-risk patients (no known cancer) with documentation of risk-reduction counseling

NCCN guideline compliance for inadequate margins requiring re-excision in BCS patients

Patients receiving antibiotics within $1 \mathrm{~h}$ before surgery

Patients receiving a first- or second-generation cephalosporin before incision

Patients with discontinuations of antibiotics within 24 h after surgery

Patients with Surgical Care Improvement Project (SCIP) antibiotic measure compliance (includes all 37 measures above)

Patients with breast cancer with documentation of risk assessment for germline mutation

Patients compliant with SCIP DVT/PE prophylaxis recommendations

Patients $\leq 50$ years with newly diagnosed breast cancer offered genetic testing

Patients presented to interdisciplinary tumor board (real or virtual) at any time

Patients compliant with NQF QM for chemotherapy in hormonal receptor-negative patients

Surgical-site infection rate (mastectomy patients)

Percentage of patients entered into a patient registry to identify patient complications and cancer outcomes 7

One-step surgery success rate stratified by type of operation (mastectomy)

Sentinel lymph node identification rate $(\%)$ in breast cancer surgery

Cosmetic score (measure of cosmesis) after BCS (patient self-assessment with Harvard score)

Time (business days) from diagnostic evaluation to needle biopsy

Time (business days) from needle biopsy path report to surgical appointment

Surgical-site infection rate (mastectomy plus plastic surgery patients)

Ipsilateral breast tumor recurrence (IBTR)

Percentage of patients undergoing lumpectomy for non-palpable cancer with two-view specimen imaging 7 performed

Percentage of compliance with ASBrS or ACR annotation of ultrasound (US) images

Percentage of compliance with ASBrS or ACR recommendations for US reports

Percentage of compliance with ASBrS or ACR recommendations for US needle biopsy reports

Compliance with ASBrS or ACR recommendations for US needle biopsy reports

NCCN guideline compliance for pre-op lab and imaging in clinical stages 0,1 , and 2 patients with cancer 7

Patients with preoperative needle biopsy proven axillary node who do not undergo sentinel node procedure

Local regional recurrence

Patients age $\geq 70$ years, hormone receptor positive, with invasive cancer offered endocrine therapy instead of radiation (documentation)

Disease-free survival

Time business days from new breast cancer to office appointment

Patients with predicted estimate of BRCA mutation $>10 \%$ who are tested

Time business days from needle biopsy path report of cancer to surgical operation

Time business days from abnormal screening mammography to diagnostic evaluation

Percentage of cancer patients entered into a quality audit (any type: institutional, personal case log, regional, national)

Time business days from new breast symptom to office appointment

Patients with benign breast disease with documentation of risk assessment for cancer

Percentage of patients with partial breast irradiation after lumpectomy who are compliant with "ASBrS 6.5
guidelines for eligibility"

Percentage of patients with partial breast irradiation after lumpectomy who are compliant with "ASTRO 6.5 guidelines for eligibility"

Number of breast-specific CMEs per year

NCCN compliance for SLN surgery in stage 0 DCIS

\begin{tabular}{|c|c|c|}
\hline 7.5 & No & Indeterminant \\
\hline 7.5 & No & Agreement \\
\hline 7 & Yes & Agreement \\
\hline 7 & Yes & Agreement \\
\hline 7 & Yes & Agreement \\
\hline 7 & Yes & Agreement \\
\hline 7 & No & Indeterminant \\
\hline 7 & No & Indeterminant \\
\hline 7 & Yes & Agreement \\
\hline 7 & No & Agreement \\
\hline 7 & No & Indeterminant \\
\hline 7 & No & Indeterminant \\
\hline 7 & No & Indeterminant \\
\hline 7 & No & Indeterminant \\
\hline 7 & Yes & Agreement \\
\hline 7 & No & Indeterminant \\
\hline 7 & No & Indeterminant \\
\hline 7 & No & Indeterminant \\
\hline 7 & No & Indeterminant \\
\hline 7 & No & Indeterminant \\
\hline 7 & No & Indeterminant \\
\hline 7 & No & Indeterminant \\
\hline 7 & No & Indeterminant \\
\hline 7 & No & Indeterminant \\
\hline 7 & No & Indeterminant \\
\hline 7 & No & Indeterminant \\
\hline 7 & No & Indeterminant \\
\hline 7 & No & Indeterminant \\
\hline 7 & No & Indeterminant \\
\hline 6.5 & No & Indeterminant \\
\hline 6.5 & No & Indeterminant \\
\hline 6.5 & No & Indeterminant \\
\hline 6.5 & No & Indeterminant \\
\hline 6.5 & No & Indeterminant \\
\hline 6.5 & No & Indeterminant \\
\hline 6.5 & No & Indeterminant \\
\hline 6.5 & No & Indeterminant \\
\hline 6.5 & No & Indeterminant \\
\hline 6.5 & No & Indeterminant \\
\hline 6.5 & No & Indeterminant \\
\hline 6.5 & No & Indeterminant \\
\hline
\end{tabular}


TABLE 2 continued

Quality domain
Median Validity $^{\mathrm{b}}$ Agreement $^{\mathrm{c}}$ score $^{\mathrm{a}}$

Skin flap necrosis rate after mastectomy stratified by type of mastectomy reconstruction, type of reconstruction

Overall survival

Ratio of malignant-to-benign minimally invasive breast biopsies

Surgical-site infection rate (all patients)

6.5 No Indeterminant

Surgeon US $(2 \times 2$ test table performance) (sensitivity, specificity, PPV, NPV) for surgeons performing 6 diagnostic breast evaluation with imaging

NCCN guideline compliance for phyllodes tumor

Compliance with ASBrS or ACR recommendations for stereotactic biopsy reports

Time business days from surgeon appointment for cancer to surgery for cancer

Percentage of mastectomy patients undergoing reconstruction

Cost of perioperative episode of care (affordability)

Patients with cancer diagnosed for core needle biopsy (CNB) for BiRads 4a lesion

Patients with cancer diagnosed for CNB for BiRads $4 \mathrm{~b}$ lesion

Patients with cancer diagnosed for CNB for BiRads $4 \mathrm{c}$ lesion

Patients with cancer diagnosed for CNB for BiRads 5 lesion

NCCN guideline compliance for Paget's disease

Surgical-site infection rate (BCS patients)

Number of axillary nodes obtained in patients undergoing level 1 or 2 nodal surgery (median)

Percentage of DCIS patients undergoing BCS for cancer who do not have axillary surgery

Patients with College of American Pathologists (CAP) compliant reporting

\section{6}

Breast cancer patients presented to interdisciplinary tumor board (real or virtual) before 1st treatment Percentage of cancer patients enrolled in clinical trials

Mastectomy patients with positive SLN who undergo completion of axillary dissection

Patients with cancer diagnosed on CNB for BiRads 3 lesion

Patients with unifocal cancer smaller than $3 \mathrm{~cm}$ who undergo BCT

Patients with documentation of pre-op breast size and symmetry

Clinical stage 0 DCIS patients who do not undergo SLN surgery for BCT

Patients undergoing level 1 or 2 axillary dissection with $\geq 15$ nodes removed

Number of SLN's (median) in patients undergoing SLN procedure

Breast volume (number of cancer cases per year per surgeon)

Percentage of cancer patients with documentation of search for clinical trial

Percentage of breast biopsy pathology requisition forms containing adequate information for pathologist 5 (history, CBE, imaging)

Time from initial cancer surgery to pathology report

Patients with documentation of pre-op contralateral breast cancer risk

Clinical stage 0 DCIS patients who do not undergo SLN surgery for mastectomy

BCT rate (actual and potential)

Time business days from abnormal screening mammogram (SM) to office appointment

Patients with documentation of needle biopsy results delivered to patients within $48 \mathrm{~h}$

BCT-eligible patients offered neoadjuvant treatment

Interval cancers (cancer detected within 1 year after negative US biopsy or stereotactic biopsy)

Cosmetic score (measure of cosmesis) after mastectomy, no reconstruction (patient self-assessment)

Percentage of cancer patients referred to medical oncology

Axillary recurrence rate

Patients with NCCN guidelines compliant care for nipple discharge

Percentage of BCT patients with marker clips placed in lumpectomy cavity to aid radiation oncologist for 5 location of boost dose for radiation

Percentage of patients with documentation of arm edema status post-operatively
6

6

6

6

\begin{tabular}{ll} 
No & Indeterminant \\
No & Indeterminant \\
No & Indeterminant \\
No & Indeterminant \\
& \\
No & Indeterminant \\
No & Indeterminant \\
No & Indeterminant \\
No & Indeterminant \\
No & Agreement \\
No & Indeterminant \\
No & Indeterminant \\
No & Indeterminant \\
No & Indeterminant \\
No & Indeterminant \\
No & Indeterminant \\
No & Indeterminant \\
No & Indeterminant \\
No & Indeterminant \\
No & Indeterminant \\
No & Indeterminant \\
No & Indeterminant \\
No & Indeterminant \\
No & Indeterminant \\
No & Indeterminant \\
No & Indeterminant \\
No & Indeterminant \\
No & Indeterminant \\
No & Indeterminant \\
No & Indeterminant \\
No & Agreement \\
\hline
\end{tabular}

No

No

No

No

No

No

No

No

No

No

No

No

Indeterminant

Indeterminant

Indeterminant

Indeterminant

Indeterminant

Indeterminant

Agreement

Indeterminant

Indeterminant

Indeterminant

Indeterminant

Disagreement

No Indeterminant

4.5

No

Indeterminant 
TABLE 2 continued

\begin{tabular}{|c|c|c|c|}
\hline Quality domain & $\begin{array}{l}\text { Median } \\
\text { score }^{\mathrm{a}}\end{array}$ & Validity ${ }^{\mathrm{b}}$ & Agreement $^{\mathrm{c}}$ \\
\hline Patients undergoing re-operation within 30 days (stratified by case type) & 4.5 & No & Indeterminant \\
\hline Patients undergoing re-admission within 30 days (stratified by base type) & 4.5 & No & Indeterminant \\
\hline Percentage of BCT patients with oncoplastic procedure performed & 4.5 & No & Indeterminant \\
\hline Patients with documentation of gynecologic/sexual side effects of endocrine therapy & 4.5 & No & Indeterminant \\
\hline Patients with documentation of gynecologic/sexual changes during follow-up & 4.5 & No & Indeterminant \\
\hline Mastectomy patients who undergo immediate intraoperative SLN assessment & 4.5 & No & Indeterminant \\
\hline Patients with latragenic injury to adjacent organ, structure (stratified by case type) & 4 & No & Indeterminant \\
\hline Percentage of lumpectomy patients with surgeon use of US intraoperatively & 4 & No & Indeterminant \\
\hline Patients with documentation of surgical pathology results delivered to patients within $96 \mathrm{~h}$ & 4 & No & Indeterminant \\
\hline Patients who have "grouped" postoperative appointments (same day, same location with care providers) & 4 & No & Indeterminant \\
\hline Percutaneous procedure complications & 3.5 & No & Indeterminant \\
\hline Percentage of patients with development of lymphedema of arm after axillary surgery & 3.5 & No & Indeterminant \\
\hline Time from initial cancer surgery to pathology report & 3 & No & Disagreement \\
\hline Patients with new DVT less than or equal to 30 days post-operatively & 3 & No & Disagreement \\
\hline Patients with new $\mathrm{PE} \leq 30$ days post-operatively & 3 & No & Indeterminant \\
\hline Documentation of use of new NSQIP-generated ACS risk calculator preoperatively & 3 & No & Indeterminant \\
\hline Patients with unplanned overnight stay stratified by procedure type & 2.5 & No & Indeterminant \\
\hline Sensitivity of immediate intraoperative detection of positive SLN (pathology quality measure) & 2.5 & No & Agreement \\
\hline Patients with myocardial infarction $\leq 30$ days postoperatively & 2 & No & Agreement \\
\hline Patients with new renal failure $\leq 30$ days postoperatively & 2 & No & Agreement \\
\hline Patients with new respiratory failure $\leq 30$ days post-operatively & 2 & No & Agreement \\
\hline
\end{tabular}

$E R$ estrogen receptor; $P R$ progesterone receptor; HER2 human epidermal growth factor 2; AJCC American Joint Committee on Cancer; NCCN National Comprehensive Cancer Network; $A D H$ Atypical Ductal Hyperplasia; ALH Atypical lobular hyperplasia; FEA Flat epithelial atypia; LCIS Lobular carcinoma in situ; MRI magnetic resonance imaging; SCIP Surgical care improvment project; $D V T$ Deep venous thrombosis; $P E$ Pulmonary embolism; ASBrS American Society of Breast Surgeons; ACR American College of Radiology; ASTRO American Society of therapuetic radiation oncologists; $C M E$ Continuing medical education credits; DCIS Ductal carcinoma in situ; $P P V$ positive predictive value; $N P V$ negative predictive value; $C B E$ clinical breast exam; NSQIP National Surgical Quality Improvement Program; ACS American Cancer Society

${ }^{\text {a }}$ Median score 1-9: lowest to highest

b Validity: $\geq 90 \%$ of the rankings are in the $7-9$ range

c Agreement: Based on scoring dispersion (e.g., for a panel of 13, there is "agreement" if $>8$ rankings are in any 3-point range and disagreement if $>3$ rankings are $1-3$ and $7-9$

Italicized text: Final ASBrS QM chosen for CMS quality payment programs

not reported here, the same process was used annually to identify new candidate QMs from 2014 to 2017 for future quality payment programs and to develop measures for the Choosing Wisely campaign. ${ }^{46}$ Based on our experience, we recommend its use for others wanting to prioritize longer lists of potential QM domains into shorter lists. These lists are iterative, allowing potential measures to be added anytime, such as after the publication of clinical trials or after new evidence-based guidelines are developed for better care. In addition, with the modified Delphi ranking process, decisions are made by groups, not individuals.

\section{After Ranking, What Next?}

Of the nine QMs selected for submission to CMS, only four had the highest possible ranking score. The reasons for not selecting some highly ranked domains of care included but were not limited to the following concerns. Some QMs were already being used by other organizations or were best assessed at the institutional, not the surgeon, level, such as the use of radiation after mastectomy for nodepositive patients. ${ }^{32-36}$ Other highly ranked measures, such as "adequate history," were not selected because they were considered standard of care.

Contralateral prophylactic mastectomy rates, a contemporary topic of much interest, was not included in our original ranking, and breast-conserving therapy (BCT) was not ranked high due to our concern that both were more a reflection of patient preferences and of regional and cultural norms than of surgeon quality. A lumpectomy reoperation QM was ranked high (7.5), but was not chosen due to disagreement within the ASBrS whether to brand 


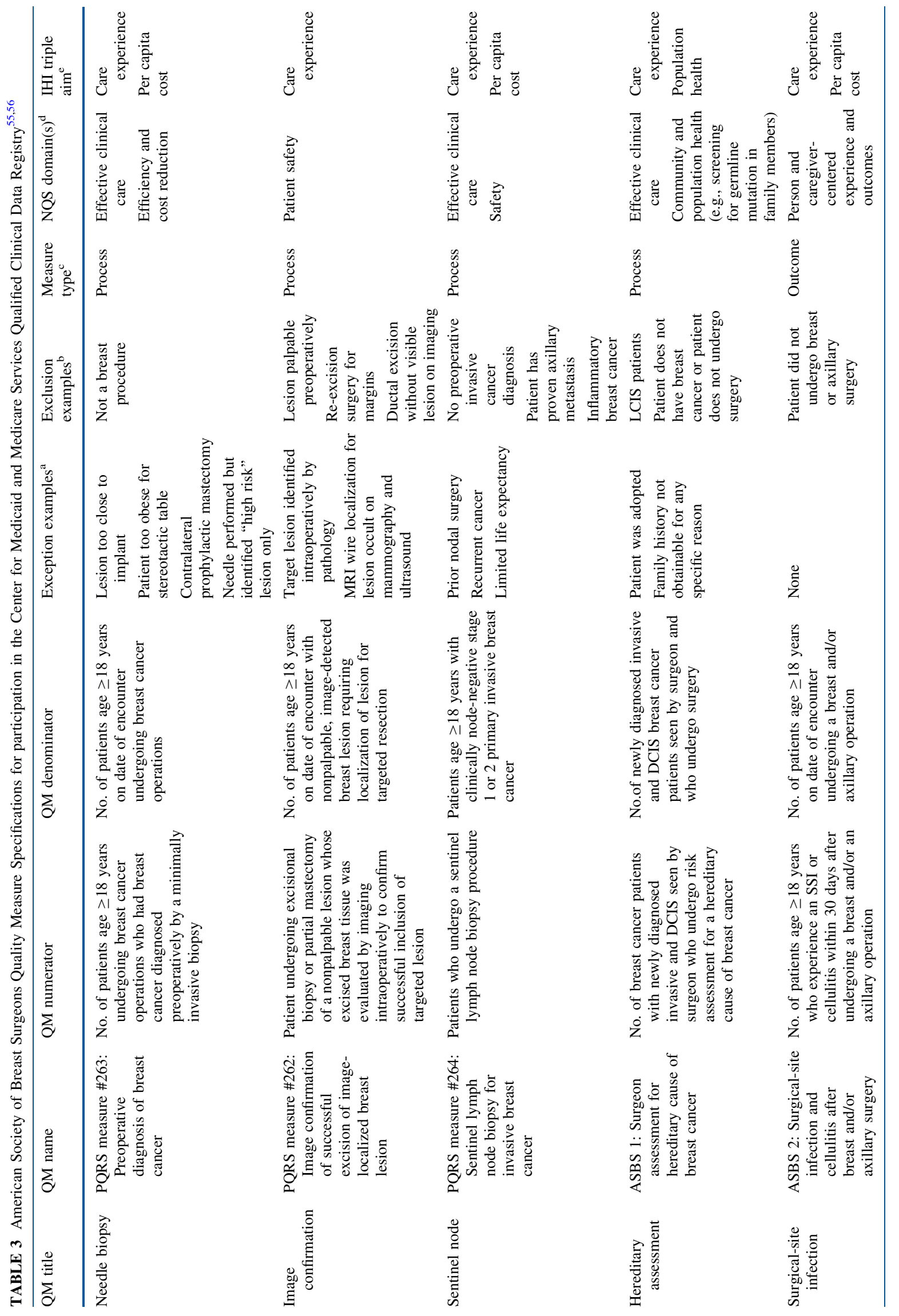









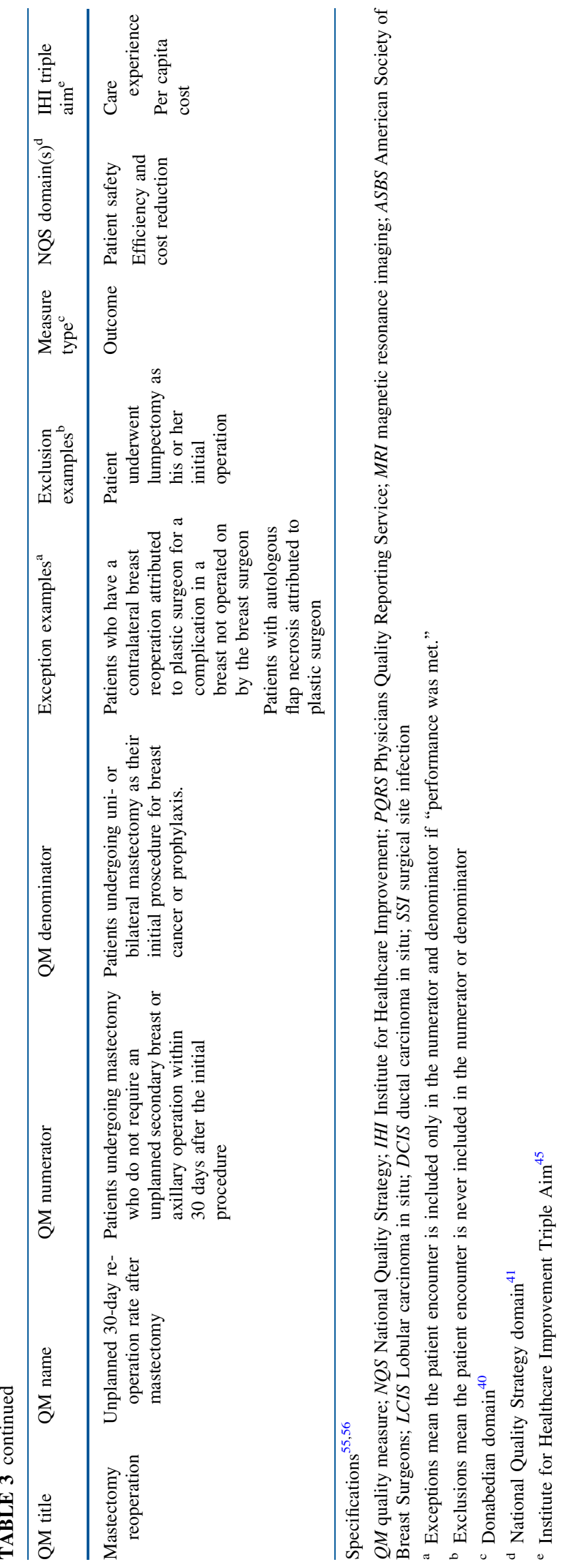

this a quality measure. ${ }^{47,48}$ In some cases, QMs with lower scores were selected for use for specific reasons. For example, by CMS rules, two QMs for a QCDR must be "outcome" measures, but all our highest ranked measures were "process of care" measures.

There was occasional overlap between our QM and those of other organizations. ${ }^{21,32-39}$ In these cases, we aimed to harmonize, not compete with existing measures. For example, a patient with an unplanned reoperation after mastectomy would be classified similarly in both the National Surgical Quality Improvement Program (NSQIP) and our program. In contrast to NSQIP, we classified a patient with postoperative cellulitis as having an SSI. Because excluding cellulitis as an SSI event has been estimated to reduce breast SSI rates threefold, adoption of the NSQIP definition would underestimate the SSI burden to breast patients and could limit improvement initiatives. ${ }^{49}$

\section{Governance}

Ranking and specifying QMs is arduous. Consensus is possible; unanimous agreement is rare. Therefore, a governance structure is necessary to reconcile differences of opinion. In our society, the PSQC solicits, ranks, and specifies QMs. A workgroup vets them for clarity and workability. In doing so, the workgroup may recommend changes. The ASBrS Executive Committee reconciles disputes and makes final decisions.

\section{Reporting Volume}

Our measurement program was successful, capturing more than 1 million provider-patient-measure encounters. On the other hand, our member participation rate was less than 20\%. By member survey (not reported here), the most common reason for not participating was "burden of reporting."

\section{Benchmarking}

"Benchmarking" is a term used most often as a synonym for peer comparison, and many programs purport to provide it. ${ }^{25}$ In actuality, benchmarking is a method for improving quality and one of nine levers endorsed by the National Quality Strategy to upgrade performance. ${ }^{21,23,30,50}$ Believing in this concept, the ASBrS and many other professional societies built patient registries that provided benchmarking. ${ }^{21,25,32-35}$ In contradistinction, the term "benchmark" refers to a point of reference for comparison. Thus, a performance benchmark can have many different meanings, ranging from a minimal quality threshold to a standard for superlative performance. ${ }^{24,36}$ 


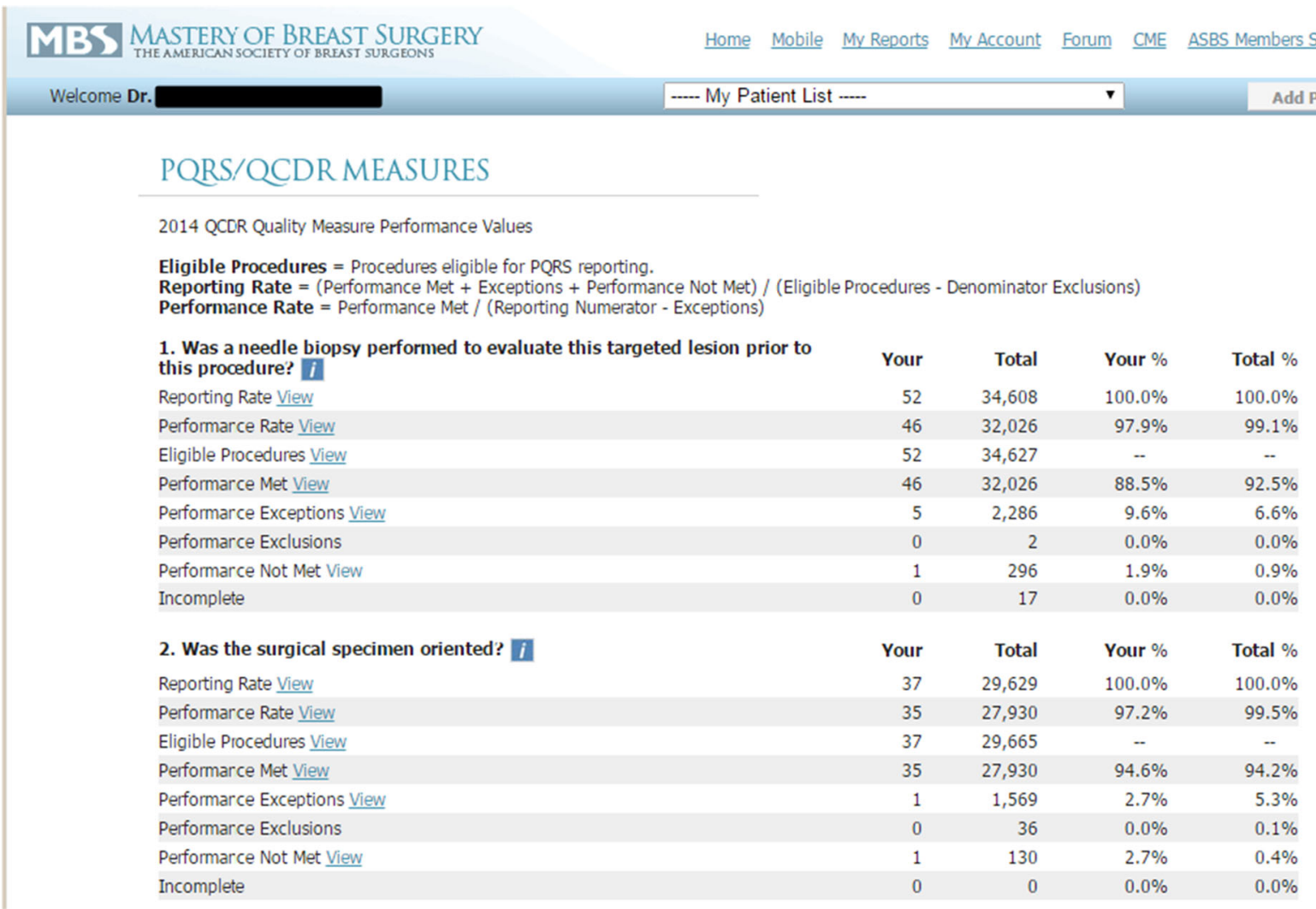

FIG. 1 Example of real-time peer performance comparison after surgeon entry of quality measures

\section{PROGRAM STRENGTHS}

Our patient registry was designed to collect specialtyspecific QMs as an alternative to adopting existing general surgical and cross-cutting measures. Cross-cutting measures, such as those that audit medicine reconciliation or care coordination, are important but do not advance specialty-specific practice. Furthermore, breast-specific measures lessen potential bias in the comparison of providers who have variable proportions of their practice devoted to the breast. Because alimentary tract, vascular, and trauma operations tend to have higher morbidity and mortality event rates than breast operations, general surgeons performing many non-breast operations are not penalized in our program for a case mix that includes these higher-risk patients. In other words, nonspecialized general surgeons who want to demonstrate their expertise in breast surgery can do so by peer comparison with surgeons who have similar case types in our program. In addition, a condition-specific program with public transparency allows patients to make more informed choices regarding their destination for care. In 2016, individual provider reportcarding for our participating surgeons began on the "physician-compare" website. ${ }^{51}$

Another strength of an organ-specific registry is that it affords an opportunity for quick Plan-Do-Study-Act
(PDSA) cycles because personal and aggregate performance are updated continuously. Thus action plans can be driven by subspecialty-specific data, not limited to expert opinion or claims data. For example, a national consensus conference was convened, in part, due to an interrogation of our registry that identified wide variability of ASBrS member surgeon reoperation rates after lumpectomy. ${ }^{52,53}$ Other program strengths are listed in Table 4.

\section{STUDY LIMITATIONS}

Although risk-adjusted peer comparisons are planned, to date, we are not providing them. In addition, only the surgeons who participate with CMS through our QCDR sign an "attestation" statement that they will enter "consecutive patients," and no current method is available for cross-checking the Mastery case log with facility case logs for completeness. Recognizing that nonconsecutive case entry (by non-QCDR surgeons) could alter surgeon performance rates, falsely elevating them, one investigation of Mastery compared the performance of a single quality indicator between QCDR- and non-QCDR-participating surgeons. ${ }^{52}$ Performance did not differ, but this analysis has not been performed for any of the QMs described in this report. Surgeons also can elect to opt out of reporting QMs at any time. The percentage of surgeons who do so 
TABLE 4 Strengths and limitations of the American Society of Breast Surgeons quality measurement program

\section{Strengths}

Specialty measures and their specifications developed by surgeons

Justifiable "exceptions" to not meeting performance defined by surgeons

Real-time surgeon data entry lessens recall bias, abstractor error, and misclassification of attribution for not meeting a performance requirement

Real-time peer performance comparison

Large sample size of patient-measure encounters $(>1,000,000)$ for comparisons

General surgeons able to compare breast surgical performance to breast-specialty surgeons

Low level of erroneous reporting based on audits

Participation satisfies American Board of Surgery Maintenance of Certification Part 4

Public transparency of individual surgeon performance in 2015 on the American Society of Breast Surgeons (ASBrS) website in 2016

Capability to use the program for "plan-do-study-act" cycles ${ }^{52,53}$

No participation fee for members before $2016^{\mathrm{a}}$

Limitations

Peer performance comparison not yet risk-adjusted

Unknown rate of nonconsecutive patient data entry

No significant patient or payer input into quality measure list or ranking to reflect their preferences and values ${ }^{54}$

Unknown rate of surgeon "dropout" due to their perception of poor performance

a $\$ 100.00$ began 2016

due to their perception of comparatively poor performance is unknown. If significant, this self-selected removal from the aggregate data would confound overall performance assessment, falsely elevating it.

Another limitation is our development of QMs by surgeons with minimal patient input and no payer input. As a result, we cannot rule out that these other stakeholders may have a perception of the quality of care delivered to them that differs from our perception. For example, patients might rank timeliness of care higher than we did, and payers of care might rank reoperations the highest, given its association with cost of care. We may not even be measuring some domains of care that are most important to patients because we did not uniformly query their values and preferences upfront during program development, as recommended by others. ${ }^{2,54}$ See Table 4 for other limitations.

\section{CONCLUSION}

In summary, the ASBrS built a patient registry to audit condition-specific measures of breast surgical quality and subsequently provided peer comparison at the individual provider level, hoping to improve national performance. In 2016, we provided public transparency of the 2015 performance reported by our surgeon participants. ${ }^{55,56}$ In doing so, we have become stewards, not bystanders, accepting the responsibility to improve patient care. We successfully captured more than 1 million patient-measure encounters, participated in CMS programs designed to link reimbursement to performance, and provided our surgeons with a method for satisfying American Board of Surgery Maintenance of Certification requirements. As public and private payers of care introduce new incentivized reimbursement programs, we are well prepared to participate with our "tested" breast-specific QMs.

ACKNOWLEDGMENT We thank Sharon Grutman for ASBrS Patient Safety and Quality Committee support, Mena Jalali for Mastery Workgroup support, Mastery Workgroup members (Linda Smith, Kathryn Wagner, Eric Brown, Regina Hampton, Thomas Kearney, Alison Laidley, and Jason Wilson) for QM vetting, Margaret and Ben Schlosnagle for quality measure programming support, Choua Vang for assistance in manuscript preparation, and the Gundersen Medical Foundation and the Norma J. Vinger Center for Breast Care for financial support. We especially thank Eric and Thomas Whitacre for Mastery program development.

DISCLOSURES No conflicts of interest to disclose.

OPEN ACCESS This article is distributed under the terms of the Creative Commons Attribution 4.0 International License (http:// creativecommons.org/licenses/by/4.0/), which permits unrestricted use, distribution, and reproduction in any medium, provided you give appropriate credit to the original author(s) and the source, provide a link to the Creative Commons license, and indicate if changes were made.

\section{REFERENCES}

1. Horwitz RI. Equity in cancer care and outcomes of treatment: a different type of cancer moonshot. JAMA. 2016 315:1231-2. doi:10.1001/jama.2016.2242. 
2. American Society of Clinical Oncology. The state of cancer care in America, 2017: a report by the American Society of Clinical Oncologists. J Oncol Pract. 2017;22:JOP2016020743. doi:10. 1200/JOP.2016.020743. Epub ahead of print.

3. Kohn LT, Corrigan J, Donaldson MS (eds). To Err Is Human: Building a Safer Health System. Institute of Medicine (US) Committee on Quality of Health Care in America. National Academies Press Washington DC, USA, 2000.

4. Institute of Medicine (USA) Committee on Quality of Health Care in America. Crossing the Quality Chasm: A New Health System for the 21st Century. National Academies Press, Washington D.C., 2001.

5. Levit LA, Balogh E, Nass SJ, Ganz PA (eds). Delivering HighQuality Cancer Care: Charting a New Course for a System in Crisis. National Academies Press, Washington, D.C., USA, 2013 (December).

6. Hewitt M, Simone JV (eds). Ensuring Quality Cancer Care. Institute of Medicine (US) and National Research Council (US) National Cancer Policy Board. National Academies Press, Washington D.C., USA, 1999.

7. Davis K, Stremikis K, Schoen C, Squires D. Mirror, mirror on the wall, 2014 update: how the U.S. health care system compares internationally. The Commonwealth Fund, 16 June 2014. Retrieved 8 December 2015 at http://www.commonwealthfund. org/publications/fund-reports/2014/jun/mirror-mirror.

8. Goodney PR, Dzebisashvili N, Goodman DC, Bronner KK. Variation in the care of surgical conditions. The Dartmouth Institute, 2015. Retrieved 8 December 2015 at http://www. dartmouthatlas.org/downloads/atlases/Surgical_Atlas_2014.pdf.

9. Balogh EP, Miller BT, Ball JR (eds). Improving Diagnosis in Health Care. Committee on Diagnostic Error in Health Care; Board on Health Care Services; Institute of Medicine; The National Academies of Sciences, Engineering, and Medicine. The National Academies Press, Washington D.C., USA, 2015 (December).

10. Agency for Healthcare Research and Quality (2016). National Healthcare Quality \& Disparities Reports. Retrieved 8 December 2015 http://www.ahrq.gov/research/findings/nhqrdr/index.html.

11. Agency for Healthcare Research and Quality (2013). 2012 National Healthcare Disparities Report. Retrieved 8 December 2015 at http://archive.ahrq.gov/research/findings/nhqrdr/nhdr12/ index.html.

12. Malin JL, Diamant AL, Leake B, et al. Quality of care for breast cancer for uninsured women in California under The Breast and Cervical Cancer Prevention Treatment Act. J Clin Oncol. 2010;28:3479-84. doi:10.1200/JCO.2009.27.7491.

13. Wilke LG, Ballman KV, McCall LM, et al. Adherence to the National Quality Forum (NQF) breast cancer measures within cancer clinical trials: a review from ACOSOG Z0010. Ann Surg Oncol. 2010;17:1989-94. doi:10.1245/s10434-010-0980-9.

14. Warner ET, Tamimi RM, Hughes ME, et al. Racial and ethnic differences in breast cancer survival: mediating effect of tumor characteristics and sociodemographic and treatment factors. $J$ Clin Oncol. 2015;33:2254-61. doi:10.1200/JCO.2014.57.1349.

15. Bekelman JE, Sylwestrzak G, Barron J, et al. Uptake and costs of hypofractionated vs conventional whole breast irradiation after breast-conserving surgery in the United States, 2008-2013. JAMA. 2014;312:2542-50. doi:10.1001/jama.2014.16616.

16. Silverstein M. Where's the outrage? J Am Coll Surg. 2009;208:78-9. doi:10.1016/j.jamcollsurg.2008.09.022.

17. Hassett MJ, Neville BA, Weeks JC. The relationship between quality, spending, and outcomes among women with breast cancer. J Natl Cancer Inst. 2014;106. doi:10.1093/jnci/dju242.

18. Greenberg CC, Lipsitz SR, Hughes ME, et al. Institutional variation in the surgical treatment of breast cancer: a study of the
NCCN. Ann Surg. 2011;254:339-45. doi:10.1097/SLA. 0b013e3182263bb0.

19. Kent EE, Mitchell SA, Castro KM, et al. Cancer care delivery research: building the evidence base to support practice change in community oncology. Clin Oncol. 2015;33:2705-11. doi:10. 1200/JCO.2014.60.6210.

20. Neuss MN, Malin JL, Chan S, et al. Measuring the improving quality of outpatient care in medical oncology practices in the United States. J Clin Oncol. 2013;31:1471-7. doi:10.1200/JCO. 2012.43.3300.

21. Cohen ME, Liu Y, Ko CY, Hall BL. Improved surgical outcomes for ACS NSQIP hospitals over time: evaluation of hospital cohorts with up to 8 years of participation. Ann Surg. 2016;263:267-73. doi:10.1097/SLA.0000000000001192.PMID: 25723845

22. Western Electric Company. Hawthorne Studies Collection, 1924-1961 (inclusive): a finding aid. Baker Library, Harvard Business School. Retrieved 9 December 2015 at http://oasis.lib. harvard.edu//oasis/deliver/deepLink?_collection=oasis\&unique $\mathrm{Id}=$ bak00047.

23. Tjoe JA, Greer DM, Ihde SE, Bares DA, Mikkelson WM, Weese JL. Improving quality metric adherence to minimally invasive breast biopsy among surgeons within a multihospital health care system. J Am Coll Surg. 2015;221:758-66. doi:10.1016/ jamcollsurg.2015.06.003. Epub 14 June 2015.

24. Kaufman CS, Shockney L, Rabinowitz B, et al. National Quality Measures for Breast Centers (NQMBC): a robust quality tool: breast center quality measures. Ann Surg Oncol. 2010;17:377-85. doi:10.1245/s10434-009-0729-5. Epub 16 October 2009.

25. Edge SB. Quality measurement in breast cancer. J Surg Oncol. 2014;110:509-17. doi:10.1002/jso.23760. Epub 27 August 2014.

26. Navathe AS, Emanuel EJ. Physician peer comparisons as a nonfinancial strategy to improve the value of care. JAMA. 2016;316:1759-60. doi:10.1001/jama.2016.13739.

27. The American Society of Breast Surgeons (2016). Mastery of Breast Surgery Program. Retrieved 10 June 2016 at https://www. breastsurgeons.org/new_layout/programs/mastery/background. php.

28. Whitacre E. The importance of measuring the measures. Ann Surg Oncol. 2009;16:1090-1. doi:10.1245/s10434-009-0370-3.

29. Laidley AL, Whitacre EB, Snider HC, Willey SC. Meeting the challenge: a surgeon-centered quality program: The American Society of Breast Surgeons Mastery of Breast Surgery Pilot Program. Bull Am Coll Surg. 2010;95:23-30.

30. Agency for Healthcare Research and Quality (2014). National quality strategy: using levers to achieve improved health and health care. Retrieved 13 June 2016 at http://www.ahrq.gov/ workingforquality/reports/nqsleverfactsheet.htm.

31. Centers for Medicare and Medicaid Services (2016). 2016 Physician Quality Reporting System (PQRS): Qualified Clinical Data Registry (QCDR). Participation made simple. Retrieved 13 June 2016 at https://www.cms.gov/Medicare/Quality-InitiativesPatient-Assessment-Instruments/PQRS/Downloads/2016PQRS_ QCDR_MadeSimple.pdf.

32. The National Accreditation Program for Breast Centers (2017). NAPBC Standards Manual, chapter on quality (chapter 6, p. 73). Retrieved 15 February 2017 https://www.facs.org/ /media/files/ quality $\% 20$ programs/napbc/2014\%20napbc\%20standards $\% 20 \mathrm{ma}$ nual.ashx\#page $=70$.

33. The American College of Surgeons (2017). Commission on Cancer Quality Measures. Retrieved 15 February 2017 at https:// www.facs.org/quality-programs/cancer/ncdb/qualitymeasures.

34. The National Consortium of Breast Centers (2017). National Quality Measures for Breast Centers. Retrieved 15 February 2017 at http:// www2.nqmbc.org/quality-performance-you-should-measure/. 
35. The American Society of Clinical Oncology (2017). Quality Oncology Practice Initiative (QOPI). Retrieved 15 February 2017 at http://www.instituteforquality.org/qopi/measures.

36. Del Turco MR, Ponti A, Bick U, et al. Quality indicators in breast cancer care. Eur J Cancer. 2010;46:2344-56. doi:10.1016/j.ejca. 2010.06.119. Epub 31 July 2010.

37. Agency for Healthcare Research and Quality (2016). The National Quality Measures Clearinghouse. Retrieved 13 June 2016 at http://www.qualitymeasures.ahrq.gov/search/search. aspx?term=breast.

38. The American Board of Internal Medicine (2016). Choosing Wisely. Retrieved 13 June 2016 http://www.choosingwisely.org/ clinician-lists/\#keyword=breast.

39. The National Quality Forum (2016). Endorsed breast cancer quality measures. Retrieved 15 February 2017 at http://www. qualityforum.org/QPS/QPSTool.aspx\#qpsPageState $=\% 7 \mathrm{~B} \% 22$ TabType $\% 22 \% 3 \mathrm{~A} 1, \% 22 \mathrm{TabContentType} \% 22 \% 3 \mathrm{~A} 1, \% 22 \mathrm{Search}$ CriteriaForStandard\%22\%3A\%7B\%22TaxonomyIDs\%22\%3A\% 5B $\% 5 \mathrm{D}, \% 22$ SelectedTypeAheadFilterOption $\% 22 \% 3 \mathrm{~A} \% 7 \mathrm{~B} \% 22$ ID $\% 22 \% 3 \mathrm{~A} 13875, \% 22$ FilterOptionLabe1\%22\%3A\%22breast $\%$ 22,\%22TypeOfTypeAheadFilterOption $\% 22 \% 3 \mathrm{~A} 1, \% 22$ Taxono myId $\% 22 \% 3 \mathrm{~A} 0 \% 7 \mathrm{D}, \% 22$ Keyword $\% 22 \% 3 \mathrm{~A} \% 22$ breast $\% 22, \% 22$ PageSize $\% 22 \% 3 \mathrm{~A} \% 2225 \% 22, \% 22 \mathrm{OrderType} \% 22 \% 3 \mathrm{~A} 3, \% 22 \mathrm{Or}$ derBy $\% 22 \% 3 \mathrm{~A} \% 22 \mathrm{ASC} \% 22, \% 22 \mathrm{PageNo} \% 22 \% 3 \mathrm{~A} 1, \% 22 \mathrm{IsExa}$ ctMatch $\% 22 \% 3$ Afalse, \%22QueryStringType $\% 22 \% 3 \mathrm{~A} \% 22 \% 22$, $\% 22$ ProjectActivityId $\% 22 \% 3 \mathrm{~A} \% 220 \% 22, \% 22 \mathrm{FederalProgram}$ Year $\% 22 \% 3 \mathrm{~A} \% 220 \% 22, \% 22 \mathrm{FederalFiscalYear} \% 22 \% 3 \mathrm{~A} \% 220$ $\% 22, \% 22$ FilterTypes $\% 22 \% 3 \mathrm{~A} 0, \% 22$ EndorsementStatus $\% 22 \% 3$ A $\% 22 \% 22 \% 7 \mathrm{D}, \% 22$ SearchCriteriaForForPortfolio $\% 22 \% 3 \mathrm{~A} \% 7$ B\%22Tags\%22\%3A\%5B\%5D,\%22FilterTypes\%22\%3A0,\%22 PageStartIndex $\% 22 \% 3 \mathrm{~A} 1, \% 22 \mathrm{PageEndIndex} \% 22 \% 3 \mathrm{~A} 25, \% 22$ PageNumber\%22\%3Anull,\%22PageSize $\% 22 \% 3 \mathrm{~A} \% 2225 \% 22, \%$ 22SortBy\%22\%3A\%22Title\%22,\%22SortOrder\%22\%3A\%22A SC $\% 22, \% 22$ SearchTerm $\% 22 \% 3 \mathrm{~A} \% 22 \% 22 \% 7 \mathrm{D}, \% 22$ ItemsTo Compare $\% 22 \% 3 \mathrm{~A} \% 5 \mathrm{~B} \% 5 \mathrm{D}, \% 22$ SelectedStandardIdList $\% 22 \%$ $3 \mathrm{~A} \% 5 \mathrm{~B} \% 5 \mathrm{D} \% 7 \mathrm{D}$.

40. Donabedian A. The quality of care: how can it be assessed? JAMA. 1988;260:1743-8.

41. Agency for Healthcare Research and Quality (2017). The National Quality Strategy Priorities. Retrieved 14 February 2017 at https://www.ahrq.gov/workingforquality/about.htm\#develnqs.

42. RAND Corporation. The RAND/UCLA Appropriateness Method User's Manual 2008. Retrieved 14 February 2017 http://www. rand.org/pubs/monograph_reports/MR1269.

43. Bilimoria KY, Raval MV, Bentrem DJ, et al. National assessment of melanoma care using formally developed quality indicators. $J$ Clin Oncol. 2009;27:5445-51. doi:10.1200/JCO.2008.20.9965. Epub 13 October 2009. Erratum in J Clin Oncol. 2010; 28:708.

44. Centers for Medicare and Medicaid Services (2016). 2016 Physician Quality Reporting System (PQRS) Toolkit: Qualified Clinical Data Registry Criteria Toolkit Measure Specifications. Retrieved 13 June 2016 https://www.cms.gov/medicare/qualityinitiatives-patient-assessment-instruments/pqrs/qualified-clinicaldata-registry-reporting.html.
45. The Institute for Healthcare Improvement (2017). Triple aim for populations. Retrieved 14 February 2017 http://www.ihi.org/ Topics/TripleAim/Pages/default.aspx.

46. Landercasper J, Bailey L, Berry T, et al. Measures of appropriateness and value for breast surgeons and their patients: the American Society of Breast Surgeons Choosing Wisely Initiative. Ann Surg Oncol. 2016;23: 3112-8. doi:10.1245/s10434-0165327-8. Epub 22 June 2016.

47. Schwartz T, Degnim AC, Landercasper J. Should re-excision lumpectomy rates be a quality measure in breast-conserving surgery? Ann Surg Oncol. 2013;20:3180-3. doi:10.1245/s10434013-3206-0. Epub 22 August 2013.

48. Morrow M, Harris JR, Schnitt SJ. Surgical margins in lumpectomy for breast cancer: bigger is not better. $N$ Engl J Med. 2012;367:79-82. doi:10.1056/NEJMsb1202521.

49. Degnim A, Throckmorton A, Boostrom S, et al. Surgical-site infection after breast surgery: impact of 2010 CDC reporting guidelines. Ann Surg Oncol. 2012;19:4099-103. doi:10.1245/ s10434-012-2448-6. Epub 26 June 2012.

50. Campion FX, Larson LR, Kadlubek PJ, Earle CC, Neuss MN. Advancing performance measurement in oncology: quality oncology practice initiative participation and quality outcomes. J Oncol Pract. 2011;7(3 Suppl):31 s-5 s. doi:10.1200/JOP.2011.000313s.

51. U.S. Centers for Medicare and Medicaid Services (2016). Physician Compare. Retrieved 14 February 2017 at https://www. medicare.gov/physiciancompare/.

52. Landercasper J, Whitacre E, Degnim AC, Al-Hamadani M. Reasons for re-excision after lumpectomy for breast cancer: insight from the American Society of Breast Surgeons Mastery(SM) database. Ann Surg Oncol. 2014;21:3185-91. doi:10.1245/ s10434-014-3905-1. Epub 22 July 2014.

53. Landercasper J, Attai D, Atisha D, et al. Toolbox to reduce lumpectomy reoperations and improve cosmetic outcome in breast cancer patients. The American Society of Breast Surgeons Consensus Conference. Ann Surg Oncol. 2015;22:3174-83. doi:10.1245/s10434-015-4759-x. Epub 28 July 2015.

54. Fayanju OM, Mayo TL, Spinks TE, et al. Value-based breast cancer care: a multidisciplinary approach for defining patientcentered outcomes. Ann Surg Oncol. 2016;23:2385-90. doi:10. 1245/s10434-016-5184-5. Epub 15 March 2016.

55. The American Society of Breast Surgeons (2016). Quality Measures and their specifications for CMS programs. Retrieved 15 February 2017 at https://www.breastsurgeons.org/new_layout/ programs/mastery/pqrs.php.

56. Centers for Medicare and Medicaid Services (2015). 2015 Physician Quality Reporting System Qualified Clinical Data Registries: The American Society of Breast Surgeons QCDR, p. 42. Retrieved 3 April 2017 at https://www.cms.gov/Medicare/ Quality-Initiatives-Patient-Assessment-Instruments/PQRS/Down loads/2015QCDRPosting.pdf.

57. The National Quality Forum (2017). Measure evaluation and importance criteria for quality measure development. Retrieved 15 February 2017 at http://www.qualityforum.org/docs/measure_ evaluation_criterias.aspx. 